\title{
Isotropic-nematic phase transition in suspensions of filamentous virus and the neutral polymer Dextran
}

\author{
Zvonimir Dogic $*$ Kirstin R. Purdy, Eric Grelet \pm Marie Adams, and Seth Fraden \\ Complex Fluids Group, Physics Department, \\ Brandeis University, Waltham, Massachusetts 02454
}

(Dated: October 8, 2018)

\begin{abstract}
We present an experimental study of the isotropic-nematic phase transition in an aqueous mixture of charged semi-flexible rods ( $f d$ virus) and neutral polymer (Dextran). A complete phase diagram is measured as a function of ionic strength and polymer molecular weight. At high ionic strength we find that adding polymer widens the isotropic-nematic coexistence region with polymers preferentially partitioning into the isotropic phase, while at low ionic strength the added polymer has no effect on the phase transition. The nematic order parameter is determined from birefringence measurements and is found to be independent of polymer concentration (or equivalently the strength of attraction). The experimental results are compared with the existing theoretical predictions for the isotropic-nematic transition in rods with attractive interactions.
\end{abstract}

PACS numbers: $64.70 . \mathrm{Md}, 64.75 .+\mathrm{g}, 61.30 . \mathrm{St}$

${ }^{*}$ current address: Rowland Institute at Harvard, Cambridge, Massachusetts 02142

${ }^{\dagger}$ permanent address: Centre de Recherche Paul Pascal, CNRS UPR 8641, Pessac, France 


\section{INTRODUCTION}

One of the fundamental notions of the theory of liquids, dating back to van der Waals, is that liquid structure is determined by the repulsive part of the intermolecular potential. The attractive part of the potential determines the density of a liquid by providing a cohesive background energy that is largely independent of a particular configuration of molecules [1, 2]. This is true as long as the liquid is far from its critical point. Due to this reason there has been a substantial effort over the past 50 years to use hard spheres as a reference system to understand the behavior of all simple liquids[3]. Parallel to these endeavors, a theory of a liquid of rods with purely repulsive anisotropic interactions was developed by Onsager 4]. It was shown that this system exhibits an isotropic-nematic (I-N) phase transition. The Onsager theory is based on the realization that the virial expansion of the free energy converges for hard rods with sufficiently large aspect ratio at the I-N phase transition, in contrast to spheres where the virial expansion fails to describe hard spheres at high concentration.

Once the behavior of a hard particle fluid is understood it is possible to study the influence of attractions via a thermodynamic perturbation theory [3, 5]. For hard spheres this is relatively easy due to the fact that attractions provide an structureless cohesive energy. In contrast, extension of the highly successful Onsager theory valid for rods with short range repulsions to a system of rods with longer range attractive interactions is much more difficult. The difficulties stem from the fact that attractive rods are in their lowest energy state when they are parallel to each other. These are exactly the configurations that need to be avoided if the second virial term on which the Onsager theory is based, is to accurately describe the system [6]. In one study the Onsager functional has been straightforwardly extended to include an additional attractive interaction 7] (from now on called Second Virial Theory with Attraction (SVTA)). Because of the problems already mentioned the author argues that SVTA is valid only for very weak attractions. Indeed, at high strengths of attraction un-physical states such as a collapse to infinitely dense state are predicted. The physical picture that emerges from the SVTA theory is that of a van der Waals like liquid of rods where its primary structure (i.e. nematic order parameter) is determined purely by the repulsive interactions, while attractions serve as a uniform structureless glue holding the rods together at a given density. 
For the theory to work at all densities the free energy of the unperturbed liquid of rods needs to take into account third and higher virial coefficients. An alternative theory that accomplished this uses scaled particle free energy of hard rods as a basis to study the influence of attractive interactions on the I-N phase transition [8, 9] (from now on called Scaled Particle Theory with Attractions (SPTA)). The scaled particle expression for hard rods includes third and higher virial coefficients. Therefore it is reasonable to expect that this theory would be more accurate at higher rod and/or polymer concentrations. An additional advantage of the SPTA theory is that it does not assume that the depletion interaction is pairwise additive. Computer simulations have shown that pairwise additivity of the intermolecular potential assumption is not an adequate approximation when the radius of the polymer is larger then the radius of the colloid [2, 10].

In this paper we experimentally study the influence of attractive interactions on the I-N transition and compare them to both SVTA and SPTA theory. As a reference system we use an aqueous suspension of semi-flexible rod-like $f d$ viruses. Previous work has shown that the behavior of $f d$ virus is consistent with the theoretical predictions for semi-flexible rods with purely repulsive interactions [11, 12, 13, 14]. Strictly speaking, $f d$ forms a cholesteric phase and undergoes an isotropic-cholesteric transition, but because the free energy difference between a cholesteric and a nematic phase is small we refer to the cholesteric phase as nematic in this paper. Additionally, introducing a finite flexibility into hard rods significantly alters both the location and nature of the isotropic-nematic and nematic-smectic phase transition [13, 15]. Here we show that flexibility also changes the isotropic-nematic phase transition in rods with attractive interactions.

We induce attraction experimentally by adding a non-adsorbing polymer to the colloidal suspensions, which leads to the depletion interaction where the range and the strength of the attractive potential is controlled by the polymer size and concentration respectively [16]. Although this work specifically deals with a colloid/polymer mixture its results are of a general significance to other anisotropic fluids which have attractive interactions due to other reasons (i.e van der Waals attractions). The main difference between polymer induced depletion attractions and attractions due to van der Waals forces is that in the depletion case there is partitioning of the polymer between coexisting phases [17]. Therefore the strength of the interaction between two colloids depends on the phase in which the colloids are located.

There have been previous experiments on the influence of polymer on the I-N phase 
transition in mixtures of boehmite rods and polystyrene polymers and mixtures of cellulose nanocrystals and Dextran polymers [18, 19, 20]. Other studies related to our work have focused on the condensation of rod-like polymers due to the presence of polymer and/or multivalent cations [21, 22]. The conditions in those studies correspond to the upper left corner of the phase diagram in Fig. 5. We also note that at very high polymer concentrations the fd system exhibits a direct isotropic-smectic coexistence and a number of metastable complex structures associated with this transition have been described elsewhere [23, 24].

In this paper we limit ourselves to the I-N transition. In Section II we present the experimental details of our measurements. In section III the effective intermolecular potential acting between two rod-like particles is discussed. In section IV we present the measured phase diagrams as a function of ionic strength and polymer size, and in section $\mathrm{V}$ we present our conclusions. In the appendix we provide the formulas necessary to calculate the phase diagrams in the SPTA theory.

\section{MATERIALS AND METHODS}

Bacteriophage $f d$ was grown and purified as described elsewhere [25]. $F d$ is a rod-like semiflexible charged polymer of length $0.88 \mu \mathrm{m}$, diameter $6.6 \mathrm{~nm}$, persistence length $2.2 \mu \mathrm{m}$ and surface charge density of $1 \mathrm{e}^{-} / \mathrm{nm}$ at $\mathrm{pH}$ 8.15. All samples where dialyzed against $20 \mathrm{mM}$ Tris buffer at $\mathrm{pH}=8.15$ and $\mathrm{NaCl}$ was added until the desired ionic strength was achieved. Dextran and FITC-Dextran with molecular weights (MW) of 500,000 and 150,000 $\mathrm{g} / \mathrm{mol}$ (Sigma, St. Louis, MO) were used as the non-absorbing polymer and dissolved in the same buffer solution. The samples are prepared in the two phase region of the phase diagram as is shown in Fig. 1. Concentrations of coexisting phases were measured using absorption spectrophotometry. The optical density of $f d$ is $\mathrm{OD}_{269}^{1 \mathrm{mg} / \mathrm{mm}}=3.84$ for a path length of $1 \mathrm{~cm}$. To determine the concentration of Dextran polymer we used a mixture of 95\% Dextran and 5\% FITC labelled Dextran. The optical density of FITC-Dextran was determined by dissolving a known amount of polymer in a buffer solution and measuring the OD at $495 \mathrm{~nm}$. The relationship between the radius of gyration $R_{q}$ of Dextran and its molecular weight $(\mathrm{MW})$ in units of $\mathrm{g} / \mathrm{mol}$ is $R_{g}[\AA]=0.66(\mathrm{MW})^{0.43}$ [26]. The reason for the small exponent 0.43 is due to the fact that Dextran is a branched polymer. The volume fraction of polymer $\phi_{\text {polymer }}$ was calculated by $\phi_{\text {polymer }}=\rho \frac{4}{3} \pi R_{g}^{3}$, where $\rho$ is the polymer 
number density. The order parameter of the nematic phase was measured with a Berek compensator, by placing the suspension into a quartz x-ray capillary with a diameter 0.7 mm (Charles Supper, Natick, MA). Samples were aligned with a 2T magnetic field [27] and the birefringence was measured. The order parameter $(S)$ is obtained using the relationship $\Delta n=S \rho_{\mathrm{fd}} n_{o}$ where $\rho_{\mathrm{fd}}$ is the number of rods per unit volume of $f d$ virus, $\Delta n$ is the birefringence measured using Berek compensator on an Olympus microscope and $\mathrm{S}$ is the nematic order which varies between 0 for the isotropic phase and 1 for a perfectly aligned phase. The birefringence of perfectly aligned $f d, n_{0}=3.8 \times 10^{-5} \pm 0.3 \times 10^{-5} \mathrm{ml} / \mathrm{mg}$, was recently obtained from x-ray experiments [28].

\section{INTERMOLECULAR POTENTIAL}

When a colloid is suspended in a polymer solution it creates around itself a shell from which the center of mass of a polymer is excluded. When two colloids approach each other there is an overlap of the excluded volume shells which leads to an imbalance of the osmotic pressure that is exerted on each colloid. This results in an effective attractive potential known as the depletion potential. In the Asakura-Oosawa model (AO), polymers are assumed to behave as spheres of radius $R^{\mathrm{AO}}$, which can freely interpenetrate each other but interact with colloids via hard core repulsive interactions [16]. The relationship between $R^{\mathrm{AO}}$ and radius of gyration of a polymer $\left(R_{g}\right)$ is $R^{\mathrm{AO}}=2 R_{g} / \sqrt{\pi}$. This approximation is valid as long as the size of the colloidal particle is much larger then the radius of the penetrable sphere $R^{\mathrm{AO}}[29,30,31]$. If the size of a colloid is equal to or smaller then $R^{\mathrm{AO}}$, the colloid can penetrate into the open polymer structure without overlapping any of the polymer segments. In this case the range and the depth of the attractive depletion potential will be significantly weaker when compared to the predictions of the AO model. In our experiments the diameter of the polymer is up to 5 times the diameter of the rod-like virus and therefore we expect that the depletion potential significantly deviates from the Asakura-Oosawa penetrable sphere model.

Since there are no analytical results on the depletion potential between rod-like colloids we estimated it using computer simulations. The method used to obtain the potential is described in detail in the paper by Tuinier et. al. 31]; here we briefly outline the procedure. Two spheres, cylinders or walls are set at a fixed distance apart and an attempt is made 
to insert a non-self-avoiding polymer molecule at random positions. When simulating the depletion potential between the cylinders they are oriented in perpendicular directions. If any segment of the polymer overlaps with either colloid, the insertion attempt fails and the polymer is not counted. The profile of the depletion potential is then equal to

$$
U_{\text {depletion }}(x)=k_{B} T(N(\infty)-N(x))
$$

where $N(x)$ is the number of polymers successfully inserted in the simulation box when two colloidal objects are a distance $x$ apart. $N(\infty)$ is the number of polymers inserted when two colloids are apart at a distance which is much larger then the range of the intermolecular potential.

The depletion potentials between walls, spheres and rods obtained from the simulations are shown in Fig. 2. From the exact results, it is known that the depletion potential at small separations between two parallel walls induced by AO penetrable spheres is equivalent to the depletion potential induced by polymer (without excluded volume interactions), if $R^{\mathrm{AO}}=2 R_{g} / \sqrt{\pi}[31,32]$. If we use this fact, the simulation results for the depletion potential between two plates (indicated by open circles in Fig. 21) are in a very good agreement with the potential predicted by the AO theory (indicated by the full line in Fig. 2), as long as the separation between the plates is smaller then $3 R_{g} / 2$. At larger separations we observe that the potential exerted by the polymer has longer range attraction than the equivalent penetrable sphere, as was previously noted [31]. This is because a polymer is only spherical on average and will adopt elongated conformations on occasion. The simulation results for the depletion potential between two spheres immersed in a polymer suspension with $R_{g} / R_{\text {colloid }}=3.36$ is significantly weaker then what is predicted by the penetrable AO sphere model. The reason for this is that a small sphere has a high probability of penetrating a polymer with a large radius of gyration since polymers have very open structures. The rods have a profile of an infinite plane in one direction and a profile of a sphere in the other direction. It follows that a cylinder with the same diameter as a sphere is less likely to interpenetrate with a polymer coil. Therefore the depletion interaction between cylinders is stronger than between spheres of equal diameter and weaker then the depletion interactions between two walls. Even for the case of cylinders, the potential obtained from the AO model significantly overestimates the strength of the potential obtained from the simulation as is shown in Fig. 2. In this paper we assume that the strength of the depletion potential between 
two cylinders oriented at an angle $\gamma$ scales as $1 / \sin \gamma$, but the shape remains independent of $\gamma$. To verify this we have simulated the potential between two cylinders that are either parallel or perpendicular to each other. For these two cases we obtain depletion potentials that are almost identical to each other after they are rescaled by a constant. This supports our assumption that the shape of the depletion potential is independent of the cylinder orientation.

The total interaction potential between two $f d$ viruses in a $f d /$ Dextran mixture is a combination of hard core repulsion, a steep short range electrostatic repulsion, and the longer range depletion attraction described above. As the ionic strength decreases both the range and the depth of the potential decreases as is shown in Fig. 3. This is due to the fact that Dextran is an uncharged polymer and therefore the depletion attraction is independent of the ionic strength. Decreasing the ionic strength results in longer range electrostatic repulsion which screens out ionic strength independent depletion attraction.

The short range electrostatic repulsion and longer range depletion attraction scale as $1 / \sin (\gamma)$ where $\gamma$ is the angle between two rods. Therefore, to a first approximation the position of the minimum of the intermolecular potential does not change when the angle between two rods changes; only the magnitude of the minimum changes. To account for the rapidly decaying electrostatic repulsion we re-scale the hard core diameter to an effective hard core diameter $D_{\text {eff }}$ as was described previously [11]. We note however that the use of $D_{\text {eff }}$ is rigourously justified only in the dilute regime where the second virial coefficient quantitatively describes the system, ie. at the isotropic-nematic transition of pure rod suspensions. Therefore one of the causes of the discrepancy between theory and experiments stems from our crude treatment of the electrostatic interactions. As discussed previously the attractive depletion potential is weaker then the predictions of the AO model. To account for this in the calculation of the phase diagram we simulated the depletion potential for experimentally relevant parameters. The simulated potential is mapped onto AO model where the effective concentrations of the interpenetrable spheres $\left(\rho_{\text {eff }}\right)$ and effective polymer radius $\left(R_{\text {eff }}^{\mathrm{AO}}\right)$ are adjusted to yield the best fit to the simulated potential. We define $\rho_{\text {eff }}=\alpha \rho$ where $\rho=N / V$ is the actual number density of $\mathrm{AO}$ penetrable hard spheres and $R_{\text {eff }}^{\mathrm{AO}}=$ $\beta R^{\mathrm{AO}}$. Surprisingly we find that the $\alpha$ is much smaller then 1 while $\beta$ is only slightly smaller than 1 for parameters used in our experiments. This can be seen in Fig. 2 where the range of the depletion potential between two spherocylinders for the AO penetrable sphere model is 
almost identical to the range of the simulated potential while the depth is very different. The reason for this is that the $\mathrm{AO}$ model underestimates the depth of the depletion attraction at large distances (Fig. 2). If the polymer size is increased further we observe that the value of $\beta$ will start decreasing rapidly. The comparison between the simulated potential and the effective potential used in the theoretical calculations of the phase diagrams is shown in the inset of Fig. 3. The phase diagrams are calculated using the effective rod diameter $D_{\text {eff }}$, the effective polymer radius $R_{\text {eff }}^{\mathrm{AO}}$, and the effective polymer concentration $\rho_{\mathrm{eff}}$. The calculation of the SPTA and SVTA phase diagrams for semi-flexible rods is described in the Appendix. Once the phase diagrams are obtained the polymer concentrations are rescaled back to the actual volume fraction of polymer. Specifically we calculate the theoretical phase diagrams using $\rho_{\text {eff }}$ and then in order to compare with experiment we plot the theoretical results using $\rho=\rho_{\text {eff }} / \alpha$.

\section{RESULTS}

In Fig. 4 a typical phase diagram for a mixture of hard rigid rods and polymers is indicated by thick full lines. As was shown in previous work by Lekkerkerker et. al. [8] adding polymer widens the isotropic-nematic coexistence and leads to partitioning of the polymer between isotropic and nematic phases. In the same figure dashed lines indicate the phase diagram of a mixture of semi-flexible rods and polymers. The influence of the flexibility on the isotropic-nematic phase transition is well studied for the case of rods with hard core repulsive interactions [33, 34]. Flexibility increases the concentration of the I-N co-existence, decreases the width of the I-N coexistence, and reduces the order parameter of the nematic phase co-existing with the isotropic phase. In Fig. 4 the theoretical phase diagrams for two equivalent systems of rods with attractions are shown with the only difference being the flexibility of the rod. For the case of the rigid rods the concentration of the polymer needed to induce widening of I-N coexisting phases is much lower than for that of semi-flexible rods. This is due to the fact that to compress semi-flexible rods, the polymer has to work against both rotational and internal bending contributions to the entropy. We conclude that flexibility also suppresses the formation of the nematic phase in attractive rods. Next we proceed to compare the theoretical phase diagrams to experiments.

A representative experimental phase diagram of an $f d$ /Dextran mixture at high ionic 
strength is shown in Fig. 5. Two features of the phase diagram are in qualitative agreement with the theoretically predicted one. First, introducing attractions widens the isotropicnematic coexistence. Second, at intermediate polymer concentrations polymer preferential partitions into the isotropic phase. At very high polymer concentration the rods and polymers are essentially immiscible with a nematic phase of pure rods coexisting with an isotropic phase of pure polymers. This part of the phase diagram has been measured elsewhere [24].

We proceed to study the influence of the ionic strength on the phase behavior. Changing ionic strength significantly modifies the interaction potential as was shown in Fig. 3. The phase diagrams at three different ionic strength are shown in Fig. 6. The experimentally measured phase diagram at $50 \mathrm{mM}$ ionic shows that the addition of the polymer has no effect on the coexistence concentrations of the I-N transition. This is in stark disagreement with theory which predicts strong partitioning of the polymer. The implication from these experimental results is that the depletion attraction is completely screened by the long range electrostatic repulsion. In calculating the potential energy between charged rods in the presence of neutral polymer we are summing two large terms of opposite signs (Fig. 3). Small inaccuracies in the theory of either of these terms could account for the discrepancy between the theoretical and experimentally observed phase diagrams.

As the ionic strength is increased to $100 \mathrm{mM}$ the addition of the polymer initially increases the width of the co-existence concentration, while at very high polymer concentration we observe re-stabilization of the I-N transition. This was also observed in mixtures of $f d$ and Dextran (MW 500,000) at $100 \mathrm{mM}$. This observation can be explained by the fact that restabilization of the I-N phase transitions occurs when the polymer is in the semi-dilute regime. In this regime the range of the depletion interaction is of the order of the correlation length (polymer mesh size), which is smaller then the radius of gyration [35]. Moreover the correlation length decreases with increasing concentration. Since the range of attraction decreases in the semi-dilute regime, the long range electrostatic repulsion will screen out any depletion attraction in the semi-dilute regime. This mechanism of depletion re-stabilization was previously observed in mixtures of charged spherical colloids and polymer mixtures in aqueous suspension [36].

At the highest ionic strength of $200 \mathrm{mM}$ a relatively low concentration of polymer is needed to induce a complete phase separation between a polymer-rich, rod-poor isotropic phase and a rod-rich, polymer-poor nematic phase. At this ionic strength no reentrant I-N 
phase behavior is observed for all accessible polymer concentrations. The phase behavior at this ionic strength has the same qualitative behavior as predicted by both SVTA and SPTA. However, when the theory is quantitatively compared to experiment there are large differences between the predicted phase boundaries (full lines) and experimentally measured phase boundaries. Perhaps the fact that the disagreement between theory and experiment is worst at low ionic strength and high rod concentration indicates that our approximation of treating electrostatically repulsive rods as hard rods with an effective diameter $D_{\text {eff }}$ is invalid under these conditions, as previously mentioned.

If attractions are introduced to a hard sphere system, the assembly will decrease its energy by decreasing the average separation between spheres, which in turn increases the density of the stable liquid phase. Unlike spheres, rod-like particles with attraction have a more complex interaction potential. They can lower their interaction energy not only by decreasing their separation (increasing their density), but also by decreasing their relative angle (increasing their order parameter), or a combination of both. To distinguish between these possibilities we measure the order parameter of the nematic phase in coexistence with the isotropic phase as is shown in Fig. 7. In Fig. 7h we compare the order parameter of rods with and without attractive interactions (ie. with and without the addition of polymer) and find that the order parameter is determined by the concentration of rods only. The nematic order parameter is plotted as a function of polymer concentration in Fig. 7b to illustrate the independence of the coexisting nematic order parameter with increasing attraction. This is further confirmed with the graph in Fig. 8, which shows that the nematic order parameter is independent of polymer concentration even well into the nematic phase. This is in agreement with both the SVTA and SPTA theories which predict that the measured order parameter of the nematic rod/polymer mixture will depend only on the concentration of rods and be independent of the level of attraction (ie. polymer concentration). We note that the order parameter data is noisy because of the intrinsic high viscosity of the fd/Dextran solutions. This high viscosity makes it difficult to create nematic monodomains even in magnetic fields up to $8 \mathrm{~T}$. 


\section{CONCLUSIONS}

We have presented quantitative measurements of the isotropic-nematic phase transition in a binary suspension of rod-like particles (fd) and spherical polymers (Dextran). The widening of the coexistence concentrations and partitioning of the polymer predicted theoretically are observed in these experiments on $f d$-Dextran mixtures. As discussed in the previous paragraph, our measurements indicate that the liquid of rods can be thought of as a van der Waals liquid where the order parameter of the nematic phase is determined by repulsive interactions, while attractive interactions provide structureless cohesive energy. Within the admittedly noisy experimental data, we find that the order parameter is determined solely by the rod concentration and not by the polymer concentration, or equivalently, the strength of attraction. However, even after taking the following effects into account; the possibility of the virus and polymer interpenetrating, the charge of the virus, and the semi-flexibility of the virus we found large quantitative differences between the theory and the experiment [37]. Notably, the theory severely overestimated the strength of the polymer induced attraction. The difference is especially pronounced in the nematic phase and at low ionic strength. This and previous work [23, 38] suggest that much remains to be done before we are able to understand and predict the behavior of rods whose interactions are more complex then simple Onsager-like hard rods.

We acknowledge the support of the National Science Foundation nsf-dmr 0088008. We thank Pavlik Lettinga for reading of the manuscript. Additional information, movies, and photographs are available online at www.elsie.brandeis.edu.

\section{APPENDIX I}

In this appendix we present the calculation of the phase diagram for a rod-polymer mixture using the SPTA theory [8, 39, 40]. Several misprints in the original paper are corrected here [8]. The approximate free energy of the colloid-polymer mixture is given by the following expression [17]:

$$
F_{C+P}(\phi)=F_{C}(\phi)-\Pi_{p}<V_{\text {free }}(\phi)>
$$

where $F_{C}(\phi)$ is the free energy of colloid suspension at volume fraction $\phi$. The co-existence 
concentrations for the I-N transition predicted by the scaled particle theory are in very close agreement with the results from the computer simulations [41]. This indicates that the scaled particles theory provides a good approximation for third and higher virial coefficients. The system is assumed to be in equilibrium with a polymer reservoir which is separated from the colloid-polymer mixture by a membrane permeable to polymers only. The osmotic pressure of the polymers in the reservoir is $\Pi_{p}=\rho k_{B} T$, with $\rho$ the polymer number density. $V_{\text {free }}$ is the free volume available to a polymer in a solution of pure hard particle colloids. It is assumed that $V_{\text {free }}$ in a polymer/colloid mixture is equal to the $V_{\text {free }}$ in the pure colloid suspension. In this sense Eq. 2 is a thermodynamic perturbation theory.

The expression for the free energy of a pure hard spherocylinder colloidal suspension is given by the scaled particle theory developed by Cotter [42] :

$$
\begin{aligned}
\frac{F_{C}(\delta, \phi, \alpha)}{N k_{b} T}= & \ln (\phi)+\ln (1-\phi)+\sigma(\alpha, L / P)+\Pi_{2}(\delta, \alpha) \frac{\phi}{1-\phi} \\
& +\frac{1}{2} \Pi_{3}(\delta, \alpha)\left(\frac{\phi}{1-\phi}\right)^{2}
\end{aligned}
$$

where $\phi$ is the volume fraction of spherocylinders

$$
\phi=\frac{N_{\text {rods }}}{V}\left(\frac{\pi}{6} D^{3}+\frac{\pi}{4} D^{2} L\right)
$$

The coefficients $\Pi_{2}$ and $\Pi_{3}$ are given by the following expressions

$$
\begin{gathered}
\Pi_{2}(\delta, \alpha)=3+\frac{3(\delta-1)^{2}}{(3 \delta-1)} \xi(\alpha), \\
\Pi_{3}(\delta, \alpha)=\frac{12 \delta(2 \delta-1)}{(3 \delta-1)^{2}}+\frac{12 \delta(\delta-1)^{2}}{(3 \delta-1)^{2}} \xi(\alpha)
\end{gathered}
$$

and the parameter $\delta$ is the overall length to diameter ratio of the spherocylinder $\delta=\frac{L+D}{D}$. The function $\sigma(\alpha, L / P)$ is an expression that accounts for the rotational entropy of the rods and the entropy associated with the loss of configurations due to confinement of the bending modes of the semi-flexible rods in the nematic phase has been derived by extrapolating between the hard rod and the flexible chain limits [43, 44, 45]. In this paper the expression for $\sigma$ obtained by Hentschke is used for numerical calculations and is given by

$$
\sigma\left(\alpha, \frac{L}{P}\right)=\ln (\alpha)-1+\pi e^{-\alpha}+\frac{L}{6 P}(\alpha-1)+\frac{5}{12} \ln \left(\cosh \left(\frac{L}{P} \frac{\alpha-1}{5}\right)\right)
$$


The function $\xi(\alpha)$ that describes the interactions between rods at the level of second virial coefficient is given by:

$$
\xi(\alpha)=\frac{2 I_{2}(\alpha)}{\sinh ^{2}(\alpha)}
$$

For this calculation we assume the Onsager ansatz for the orientational distribution function given by :

$$
f(\alpha, \cos (\theta))=\frac{\alpha \cosh (\alpha \cos (\theta))}{4 \pi \sinh (\alpha)} .
$$

The expression for the free volume in a spherocylinder suspension is given by:

$$
\nu(\phi, \delta, q)=\frac{V_{\text {free }}}{V}=(1-\phi) \exp \left(-\left(A(\delta, q)\left(\frac{\phi}{1-\phi}\right)+B(\delta, q)\left(\frac{\phi}{1-\phi}\right)^{2}+C\left(\frac{\phi}{1-\phi}\right)^{3}\right)\right)
$$

where

$$
\begin{aligned}
A(\delta, q) & =\frac{6 \delta}{3 \delta-1}+\frac{3(\delta+1)}{3 \delta-1} q^{2}+\frac{2}{3 \delta-1} q^{3} \\
B(\delta, q) & =\frac{1}{2}\left(\frac{6 \delta}{3 \delta-1}\right)^{2} q^{2}+\left(\frac{6}{3 \delta-1}+\frac{6(\delta-1)^{2}}{(3 \delta-1)^{2}} \xi(\alpha)\right) q^{3} \\
C(\delta, q) & =\frac{24 \delta}{3 \delta-1}\left(\frac{2 \delta-1}{(3 \delta-1)^{2}}+\frac{(\delta-1)^{2}}{(3 \delta-1)^{2}} \xi(\alpha)\right) q^{3}
\end{aligned}
$$

The ratio of the polymer diameter to the rod diameter is given by the parameter $q$. After the expression for the scaled particle free energy ( 3) is obtained, we insert the Onsager approximation for the orientational distribution functions $f(\alpha)$ and minimize the free energy at different rod concentrations with respect to the parameter $\alpha$ to find the order parameter of the nematic phase at that concentration. To find the concentrations of rods in the coexisting isotropic and nematic phases we solve the conditions for the equality of the osmotic pressure and chemical potential. The expressions for the osmotic pressure and the chemical potential are :

$$
\begin{gathered}
\Pi=\phi^{2} \frac{\partial F_{c}(\phi)}{\partial \phi}+n_{p} \lambda\left(\nu-\phi \frac{\partial \nu(\phi)}{\partial \phi}\right) \\
\mu=F_{c}(\phi)+\phi \frac{\partial F_{c}(\phi)}{\partial \phi}+n_{p} \lambda \frac{\partial \nu(\phi)}{\partial \phi}
\end{gathered}
$$


where $n_{p}$ is the polymer volume fraction, $\frac{4}{3} \pi R_{g}^{3} \rho$, and $\lambda$ is the ratio of spherocylinder volume to polymer volume

$$
\lambda=\frac{1}{q^{3}}\left(1+\frac{3}{2}(\delta-1)\right)
$$

The phase diagram is calculated by first minimizing the free energy with the respect to the parameter $\alpha$ and then solving coexistence equations( 12). The SVTA phase diagrams for rigid rods are calculated following the calculation of Warren [7]. To extend this calculation to semi-flexible rods the orientational entropy term in the Onsager free energy was replaced by the confinement entropy of semi-flexible polymers as shown in Eq. 7. To account for electrostatics the rod diameter $D$ is replaced with $D_{\text {eff }}[1]$. To correct the AO theory for the case of a polymer radius larger than the colloid radius we replace the polymer density $\rho$ with $\rho_{\text {eff }}$ and the polymer radius $R_{g}$ with $R_{\text {eff }}^{\mathrm{AO}}$ as described in the text and in Figures 3,5 and 6 .

[1] B. Widom, Science 157, 375 (1967).

[2] W. M. Gelbart and B. Barboy, Acc. Chem. Res. 13, 290 (1980).

[3] J. P. Hansen and I. R. McDonald, Theory of Simple Liquids (Academic Press, London:, 1986), 2nd ed.

[4] L. Onsager, Ann. NY Acad. Sci. 51, 627 (1949).

[5] A. P. Gast, W. B. Russel, and C. K. Hall, J. Colloid Interface Sci. 109, 161 (1986).

[6] P. van der Schoot and T. Odijk, J. Chem. Phys. 97, 515 (1992).

[7] P. B. Warren, J. Phys. I (France) 4, 237 (1994).

[8] H. N. W. Lekkerkerker and A. Stroobants, Nuovo Cimento D 16, 949 (1994).

[9] P. G. Bolhuis, A. Stroobants, D. Frenkel, and H. N. W. Lekkerkerker, J. Chem Phys 107, 1551 (1997).

[10] E. J. Meijer and D. Frenkel, J. Chem. Phys. 100, 6873 (1994).

[11] J. Tang and S. Fraden, Liquid Crystals 19, 459 (1995).

[12] S. Fraden, in Observation, Prediction, and Simulation of Phase Transitions in Complex Fluids, edited by M. Baus, L. F. Rull, and J. P. Ryckaert (Kluwer Academic Publishers, 1995), pp. $113-164$.

[13] Z. Dogic and S. Fraden, Phys. Rev. Lett. 78, 2417 (1997). 
[14] Z. Dogic and S. Fraden, Langmuir 16, 7820 (2000).

[15] A. R. Khokhlov and A. N. Semenov, Physica 108a, 546 (1981).

[16] S. Asakura and F. Oosawa, J. Polym. Sci. 33, 183 (1958).

[17] H. N. W. Lekkerkerker, W. C.-K. Poon, P. N. Pusey, A. Stroobants, and P. B. Warren, Europhys. Lett. 20, 559 (1992).

[18] J. Buitenhuis, L. N. Donselaar, P. A. Buining, A. Stroobants, and H. N. Lekkerkerker, J. Colloid Interface Sci. 175 (1995).

[19] M. P. B. van Bruggen and H. N. W. Lekkerkerker, Macromolecules 33, 5532 (2000).

[20] C. D. Edgar and D. G. Gray, Macromolecules 35, 7400 (2002).

[21] J. X. Tang, S. Wong, P. T. Tran, and P. A. Janmey, Ber. Bunsenges. Phys. Chem. 100, 796 (1996).

[22] R. de Vries, Biophy. J. 80, 1186 (2001).

[23] Z. Dogic and S. Fraden, Phil. Trans. R. Soc. Lond. A. 359, 997 (2001).

[24] Z. Dogic, Phys. Rev. Lett. 91, 165701 (2003).

[25] T. Maniatis, J. Sambrook, and E. F. Fritsch, Molecular Cloning (Cold Spring Harbor University Press, 1989).

[26] F. R. Senti, N. N. Hellman, N. H. Ludwig, G. E. Babcock, R. Tobin, C. A. Glass, and B. L. Lamberts, J. Poly. Sci. 17, 527 (1955).

[27] R. Oldenbourg and W. C. Phillips, Rev. Sci. Instrum. 57, 2362 (1986).

[28] K. R. Purdy, Z. Dogic, S. Fraden, A. Ruhm, L. Lurio, and S. G. J. Mochrie, Phys. Rev. E 67, $031708(2003)$.

[29] A. P. Chatterjee and K. S. Schweizer, J. Chem. Phys. 109, 10477 (1998).

[30] A. Hanke, E. Eisenriegler, and S. Dietrich, Phys. Rev. E. 59, 6853 (1999).

[31] R. Tuinier, G. A. Vliegenthart, and H. N. W. Lekkerkerker, J. Chem. Phys. 113, 10768 (2000).

[32] S. Asakura and F. Oosawa, J. Chem. Phys. 22 (1954).

[33] G. J. Vroege and H. N. W. Lekkerkerker, Repts. on Prog. Phys. 8, 1241 (1992).

[34] Z. Y. Chen, Macromolecules 26, 3419 (1993).

[35] R. Verma, J. C. Crocker, T. C. Lubensky, and A. G. Yodh, Macromolecules 33, 177 (2000).

[36] A. P. Gast, C. K. Hall, and W. B. Russel, Farday. Discuss. Chem. Soc. 76, 189 (1983).

[37] R. P. Sear, Phys. Rev. E 55, 5820 (1997).

[38] M. Adams, Z. Dogic, S. L. Keller, and S. Fraden, Nature 393, 349 (1998). 
[39] H. N. W. Lekkerkerker, in Structure and Dynamics of Strongly Interacting Colloids and Supramolecular Aggregates in Solution, edited by S. H. Chen, et.al. (Kluwer Academic Publishers, 1992), p. 97.

[40] E. J. Meijer and D. Frenkel, Phys. Rev. Lett. 67, 1110 (1991).

[41] E. M. Kramer and J. Herzfeld, Phys. Rev. E 58, 5934 (1998).

[42] M. A. Cotter, in The Molecular Physics of Liquid Crystals, edited by G. R. Luckhurst and G. W. Gray (Academic Press, London, 1979), pp. 169-189.

[43] R. Hentschke, Macromolecules 23, 1192 (1990).

[44] T. Odijk, Macromolecules 19, 2313 (1986).

[45] D. P. DuPre and S. Yang, J. Chem. Phys. 94, 7466 (1991). 


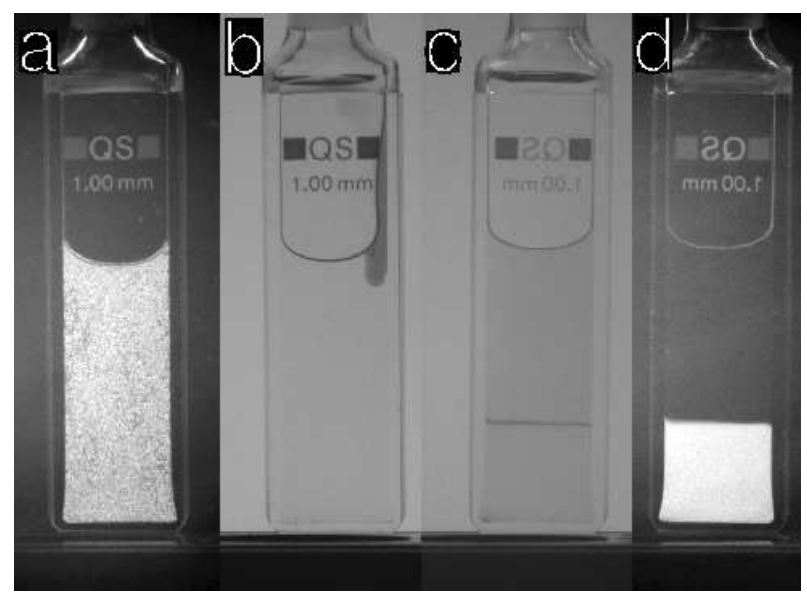

FIG. 1: (Color online) A sequence of images illustrating the preparation of a sample which was used in determining the phase diagram. a) A nematic liquid crystal of $f d$ virus in buffer between crossed polarizers showing disordered birefringent domains. b) A highly concentrated solution of Dextran labelled with yellow fluorescein is added to the transparent $f d$ nematic liquid crystal. c) After the sample in (b) is vigorously shaken it phase separates into the coexisting nematic and isotropic phases. The macroscopic phase separation takes from few hours to couple of days depending on the location in the phase diagram. d) Same sample as image (c) but taken between crossed polarizers. Image shows dense birefringent nematic phase on the bottom and Dextran rich isotropic phase on the top which is yellow in appearance. Images (a) to (d) are taken on the same sample. 


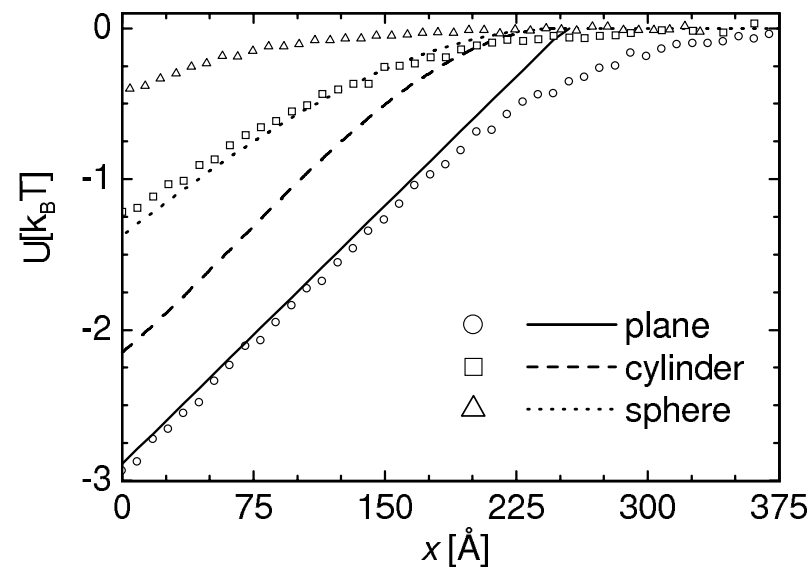

FIG. 2: Depletion potential $U$ between two walls, two perpendicular cylinders and two spheres obtained from computer simulation are shown by open spheres, squares and triangles respectively. In the two wall simulation the wall size was $313 \times 313 \AA^{2}$ and periodic boundary conditions where used. The diameter of the spheres and cylinders is $66 \AA$ while $R_{g}$ of the polymer is $111 \AA$. The lines indicate depletion potentials as predicted by the penetrable sphere (AO) model. The separation $x$ is the closest distance between two surfaces. The number concentration of the polymer $\rho$ is equal to the overlap concentration $\rho=3 /\left(4 \pi R_{g}^{3}\right)$, while the radius of the penetrable spheres is $R^{\mathrm{AO}}=2 R_{g} / \sqrt{\pi}=125 \AA$. The $\mathrm{AO}$ theory overestimates the potential between spheres and between cylinders because polymer deform around colloidal particles. 


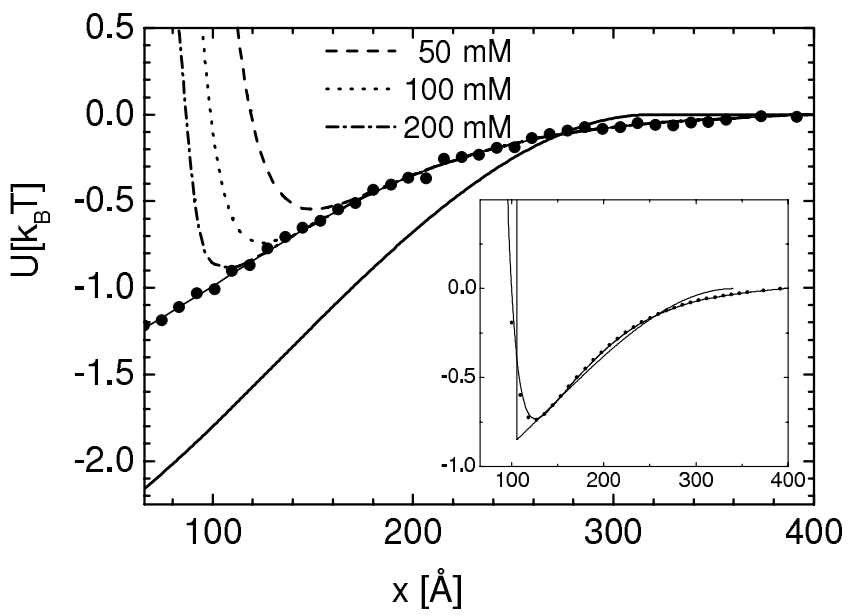

FIG. 3: Total interaction potential $U$ as a function of separation $x$ between two viruses $(D=$ $66 \AA$ ) oriented at $90^{\circ}$ with respect to each other and immersed in a suspension of polymers of concentration $\rho=3 /\left(4 \pi R_{g}^{3}\right)$ and radius $R_{g}=111 \AA$ at three different ionic strengths. The interaction potential is a sum of electrostatic repulsion and depletion interaction. The effect of electrostatic repulsion for $f d$ with net linear charge density $1 e^{-} / \AA$ A of is accounted for by treating the the $f d$ as a hard particle with a larger effective diameter $D_{\text {eff }}[11$, 33]. Filled circles indicate the depletion potential obtained from Monte Carlo simulation of polymers without excluded volume interactions. Since the polymer diameter is larger then the rod diameter, the polymer and rodlike viruses can easily interpenetrate. This results in a effective depletion attraction which is smaller than what is predicted by the AO model (indicated by the full line). The phase diagrams corresponding to these interaction potentials are shown in Fig. 6. Inset : In theoretical calculations we approximate the intermolecular potential between rods with an effective hard core diameter $D_{\text {eff }}$ 11] and attractive potential. The attractive part of the potential is modeled by AO penetrable spheres whose effective radius and concentration best fits the potential obtained through computer simulation. This effective intermolecular potential is compared to the potential obtained through the computer simulation in the inset. In the inset $\rho_{\text {eff }} / \rho=0.39$ and $R_{\text {eff }}^{\mathrm{AO}} / R^{\mathrm{AO}}=0.99$. 


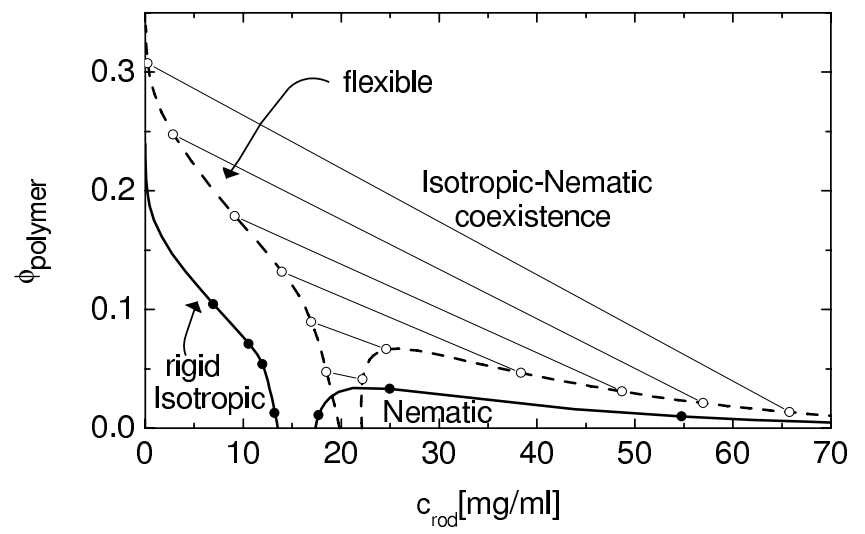

FIG. 4: Phase diagram for rigid and semi-flexible rods calculated using the SPTA theory. The boundary between the isotropic(I)-nematic $(\mathrm{N})$ two phase region and the region where a single phase is stable is indicated by the thick dashed line for semi-flexible rods and thick full lines for rigid rods. Tie lines between the coexisting phases are shown by thin lines. For the flexible particle the ratio of the contour length to persistence length is $L / P=0.4$. The phase diagram was calculated using $\delta=84$ and $q=2.2$. The polymer concentration is defined as follows $\phi_{\text {polymer }}=\rho \frac{4 \pi R_{g}^{3}}{3}$.

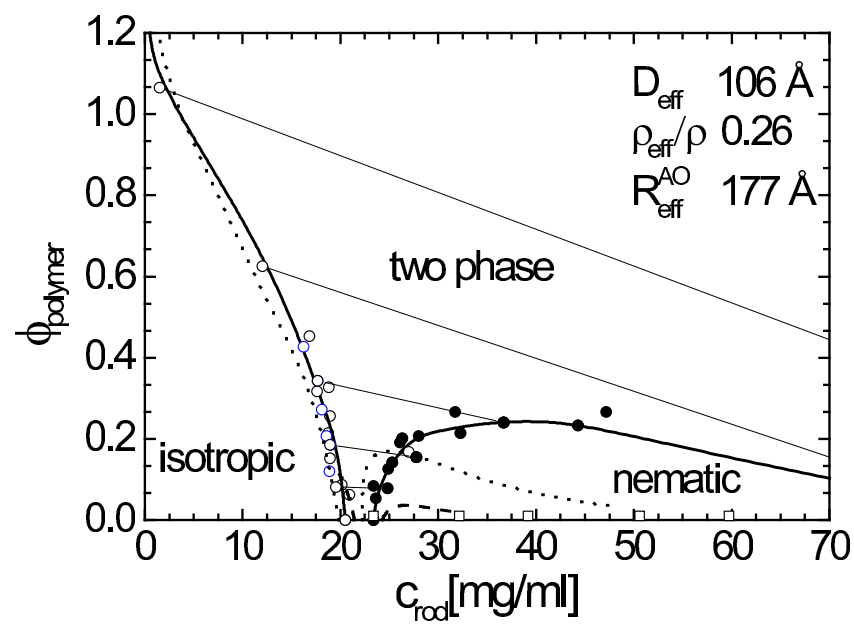

FIG. 5: Phase diagram for a mixture of $f d$ virus and Dextran (MW 500,000, $R_{g}=176 \AA$ or $\left.R^{\mathrm{AO}}=199 \AA\right)$ at $100 \mathrm{mM}$ ionic strength. The measured points indicate the rod and polymer concentrations of the coexisting isotropic and nematic phases. The full line is a guide to the eye indicating the two phase region. Tie-lines are indicated by thin full lines. The SPTA and SVTA predictions are indicated by the dotted lines and dashed lines respectively. 


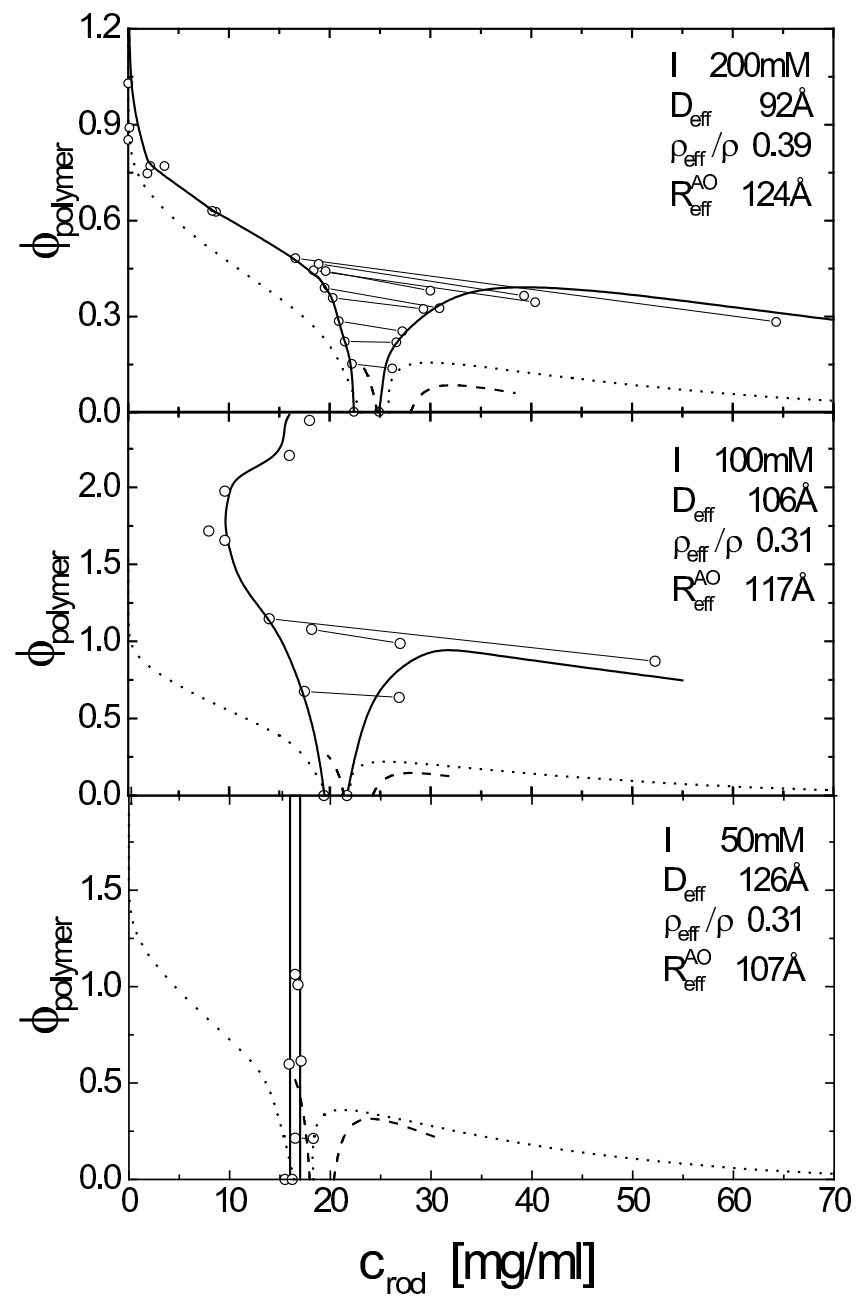

FIG. 6: Phase diagrams of a mixture of $f d$ virus and Dextran polymer (MW 150,000, $R_{g}=111 \AA$ or $R^{\mathrm{AO}}=125 \AA$ ) at $50 \mathrm{mM}, 100 \mathrm{mM}$ and $200 \mathrm{mM}$ ionic strength. Coexisting phases are indicated by open circles while the full line is an eye guide separating two phase region from isotropic and nematic phases. The predictions of the SPTA and SVTA theories are indicated with dashed and dotted lines respectively. The polymer concentration is defined as follows $\phi_{\text {polymer }}=\rho \frac{4 \pi R_{g}^{3}}{3}$. 

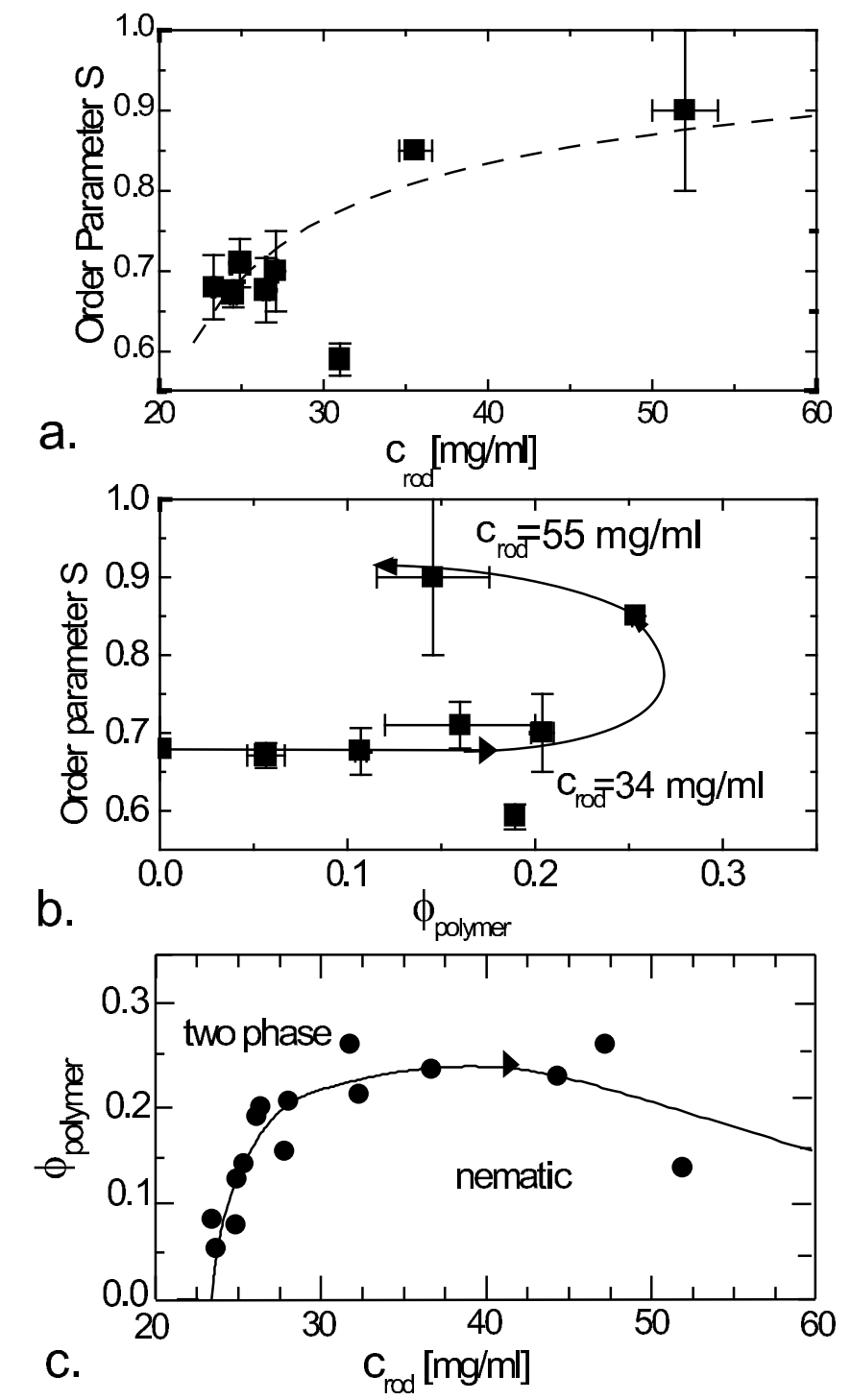

FIG. 7: Measurements of the order parameter of the nematic phase in coexistence with the isotropic phase for a mixture of $f d$ (rod) and Dextran (M.W. 500,000, polymer) at $100 \mathrm{mM}$ ionic strength. Order parameter (S) is graphed as a function of $f d$ concentration (a) and Dextran concentration (b) for the coexisting nematic concentrations shown in (c). The order parameter is double valued in (b) because along the nematic branch of the coexistence curve there are two different rod concentrations with the same polymer concentration as shown in (c). Data in (c) is the same as that shown in Fig. 5. Dashed line in (a) indicates the theoretical dependence of the order parameter on the concentration of rods as obtained using scaled particle theory. This relationship agrees well with experimental data for $f d$ at high ionic strength using x-ray scattering 28]. Arrows in (b) and (c) indicate the direction of increasing $f d$ concentration. Below $\phi_{\text {polymer }} \sim 0.2$ the nematic $f d$ concentration is essentially constant. 


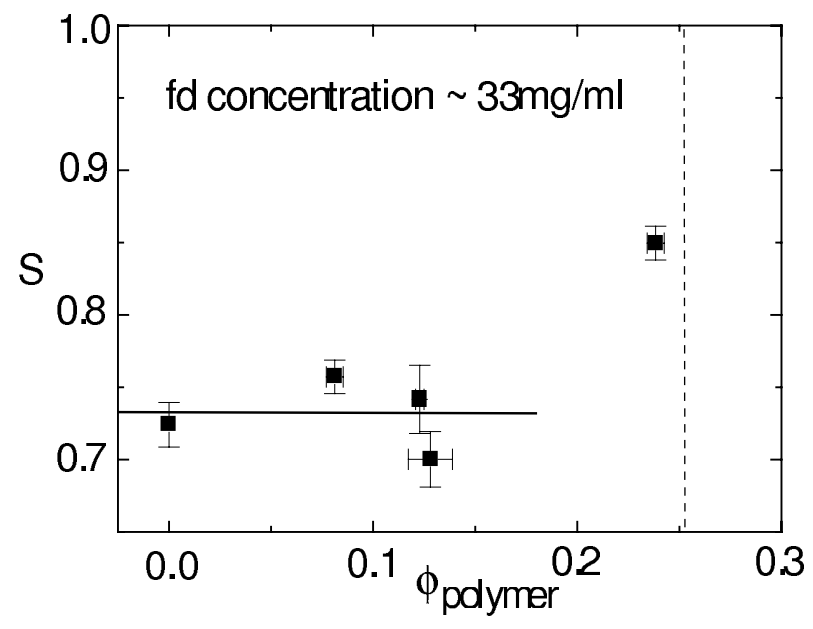

FIG. 8: The order parameter of the nematic phase of the $f d /$ Dextran (M.W. 500 000) mixture at $33 \mathrm{mg} / \mathrm{ml} f d$ and $100 \mathrm{mM}$ ionic strength as a function of increasing polymer concentration. The horizontal line drawn is a guide to the eye showing the independence of the nematic order parameter with polymer concentration. The vertical line indicates the location of the nematicisotropic transition. 